\title{
Opportunities and Challenges of AC/DC Transmission Network Planning Considering High Proportion Renewable Energy
}

\author{
Arslan Habib 1*, Chan Sou 2, Adeel Arshad ${ }^{3}$, Hafiz Muhammad Hafeez ${ }^{4}$ \\ 1 School of Automation, Northwestern Polytechnical University China, CHINA \\ 2 State Grid Tianjin Energy Saving Service Co., Ltd, CHINA \\ ${ }^{3}$ Fluids \& Thermal Engineering (FLUTE) Research Group, University of Nottingham, UK \\ ${ }_{4}^{4}$ School of Mechanical Engineering, Northwestern Polytechnical University, CHINA
}

*Corresponding Author: arslan.2010@live.com

Citation: Habib, A., Sou, C., Arshad, A. and Hafeez, H. M. (2018). Opportunities and Challenges of AC/DC Transmission Network Planning Considering High Proportion Renewable Energy. European Journal of Sustainable Development Research, 2(2), 17. https://doi.org/10.20897/ejosdr/83707

Published: March 8, 2018

\begin{abstract}
The time and space distribution characteristics of future high proportion of renewable energy sources will bring unprecedented challenges to the electric power system's processing and planning, the basic form of electric power system and operating characteristics will have fundamental changes. Based on the research status quo at home and abroad, this paper expounds the four scientific problems of the transmission network planning with high proportion of renewable energy. Respectively, from the network source collaborative planning, transmission network flexible planning. With the distribution network in conjunction with the transmission network planning, transmission planning program comprehensive evaluation and decision-making methods. This paper puts forward the research ideas and framework of transmission network planning considering the high proportion of renewable energy. At the end, the future high proportion of (renewable energy) grid-connected transmission network's opportunities and challenges are presented.
\end{abstract}

Keywords: flexible planning, comprehensive evaluation, high proportion of renewable energy, transmission network planning, network source coordination

\section{INTRODUCTION}

21st century's energy security and climate change are two major challenges facing human society. Vigorously develop wind power, Solar energy, hydropower and other renewable energy, are the need to time to achieve energy and economic sustainable development (Energy Research Institute, 2015; Hand et al., 2014; Price Water House Coopers LLP, 2014; Lu et al., 2016). In the current renewable energy grid connected scenarios, the system has been seriously abandoned. For example, in china "The national wind power operation" pointed out that in the first half of the year 2016, wind power 32.3 TW.h was abandoned, and the increase was of 14.8 TW.h, the average rate of abandoned $21 \%$, an increase of 6 percent. In the first half of 2016, the average utilization rate of wind power was 917 hours, down by 85 hours. Abandoning the wind and abandoning the light have greatly hindered the healthy and sustainable development of China's renewable energy resources. To solve the problem of renewable energy has become the major need of the country.

One of the most important principles of power grids is that they always meet the power requirements and equal the electricity consumption, the fluctuation of the network load leads to the dynamic balance between the power generation level and the power load, namely the stability of the grid, and the grid itself does not store any power. 
Today's power grid does not have the flexibility to transmit a large amount of scattered and diversified renewable energy. These two features of renewable energy directly impede the integration of new energy into the grid. In March 2016, the guidance issued by the National Energy Administration 'Guiding Opinions on Establishing the Guiding system for the Development and Utilization of Renewable Energy Resources' further strengthened the position of renewable energy in China's power system (Arslan et al., 2017).

For the transmission network, the high proportion of renewable energy access will complicate the operation of the power system and complicate the AC / DC connection of the power grid (Kang and Yao, 2017). Although there is are certain research foundations for the transmission network planning of renewable energy sources in the world, there is still a lack of research on the impact of high proportion renewable energy on the planning, mechanism research and mature planning methods. This paper focuses on the transmission network planning of high proportion renewable energy sources. Firstly, on the basis of full investigation and study of the current situation, the scientific problems faced by the transmission network planning under high proportion renewable energy resources are put forward. Then, the research framework of transmission network planning theory is expounded from the following three aspects: (1) Network source collaborative planning method; (2) Transmission network flexible planning method; (3) with the distribution network in conjunction with the transmission network planning method. Then, in view of the complexity of the planning decision making for the transmission network planning with high proportion of renewable energy sources, the comprehensive evaluation and decision-making methods of the planning scheme based on security check and production simulation fusion technology are expounded. Finally, the prospect and summary of the transmission network planning for high proportion renewable energy.

\section{RESEARCH STATUS QUO}

The transmission network is connected with the power supply and the load, the planning decisions directly affect renewable energy consumption, is a very important basic work in power system (Hemmati et al., 2013; Latorre et al., 2003). In the context of high proportion renewable energy access, the theory and method of transmission network planning need more research.

From the point of view of network source coordination, power grid planning and power planning are largescale, high dimension and nonlinear mixed integer programming problems, and optimization calculation is very difficult. In the traditional power planning, the source uncertainties are relatively few, so the power planning and power grid planning can be solved separately. With the continuous infiltration of the strong and uncertain renewable energy sources, the uncoordinated and mismatched optimization of the power supply and the grid makes the problem of abandoning the air and abandoning the light increasingly prominent (Guy et al., 20012), It is necessary to study the network source collaborative planning method with high proportion of renewable energy. The existing literature builds the network source collaborative planning model based on different angles. For example: the literature (Rouhani et al., 2014) proposed a distributed power source considering the network source collaborative planning model, and in the actual large-scale system testing; literature (Aghaei et al., 2014) the reliability index into the network source collaborative planning model; In (Gao et al., 2012), a network source collaborative programming model is proposed to consider the combination of regulator and grid planning. In (Zhang et al., 2016), a covariant cohort programming model with both randomness and ambiguity of wind power is proposed. However, there is a lack of research on the network source co-planning for the future consideration of the spatial and temporal distribution of high proportion of renewable energy.

From the perspective of flexible planning, access to high proportion of intermittent renewable energy will give the grid more uncertainty. The flexible planning method takes into account the uncertainties in the planning process in order to find a flexible planning scheme with better flexibility and adaptability (Cheng et al., 2007).

MAS (Multi-Agent System) is a kind of artificial intelligence computing method; its independent characteristics make it possible for electronic devices with common or conflicting goals to coexist in complex power systems. MAS based on JADE (Java Agent Development Framework) platform for Agent information interactive, distributed simulation based on JADE simulation platform has become an important research direction in recent years (Habib et al., 2017).

At present, in network planning uncertainty mainly has two kinds of methods: stochastic programming (Alvarez et al., 2007; Majidi-Qadikolai and Baldick, 2016; Akbari et al., 2011; Roh et al., 2009; Carrion et al., 2007; Orfanos and Georgilakis, 2013) and robust optimization method (Chen and Wang, 2016; Chen et al., 2014; Ruiz and Conejo, 2015). Transmission network stochastic programming research is more mature. Literature (Yuan et al., 2009) apply the method of multi scenario probability to consider the uncertain factors such as wind power output and load change, and put forward the planning method of transmission system taking into account large-scale wind farm; Literature (Zhou et al., 2011) uses a field based on extreme scene sets the scene method replaces the scene method based on Monte Carlo simulation, and improves both the computation time and the precision. Literature (Ding et 
al., 2012) describes the uncertainty of wind power by the certainty of multiple scenarios, and gives the cost of line investments in planning scenarios based on constraints such as DC power flow, Optimal power flow calculation model with generation cost, outage cost and load minimization as objective function. Document (Yu et al., 2009) based on wind speed distribution and wind power curve of wind-driven generator, the probability distribution function and probability density function of wind power generator are derived. In (Huang et al., 2013), the influence of the power generation scheme on the transmission network is analyzed and considered without considering the correlation between the output of the wind farm, on the basis of this, a two-layer model of transmission network planning considering the capacity of intermittent power supply is constructed. Transmission network planning based on multi scene technology is the mainstream method at present, but there are still many problems such as large-scale problem solving, low frequency event, random characteristics difficult to obtain and so on. Different from the stochastic programming method using robust optimization method in transmission network planning is still in the initial stage of research, which does not require the probability distribution of uncertain parameters, solving scale is relatively small compared with the stochastic programming. Literature (Jabr, 2013) proposes a robust planning method for transmission network considering the uncertainty of output and load of renewable energy. The uncertainty set is constructed to describe the uncertainty factors. The robust programming method can resist the bad scene, but the planning scheme is too conservative, Adaptive robust optimization (Dehghana et al., 2017), light robust optimization (Tan et al., 2016) and so on are one of the approaches to solve the problem of conservatism, Literature (Zhao and Guan, 2013) combines robust optimization with stochastic programming to improve conservatism.

Energy storage devices are arranged in the non-grid-connected wind power generation system to ensure the power quality, and it has become the key to full utilization of renewable energy. In the case of wind speed fluctuation, the DC bus control strategy of the wind turbine is proposed in this reference. It can reduce the impact on the unit converter and the power load; this ensures safe and stable operation of non-grid connected wind turbines (Habib et al., 2017).

From the perspective of transmission and distribution grid coordination, the degree of coordination of transmission and distribution network is an important basis for power system security and economy. To ensure the full coordination of the voltage level of the grid, neither the formation of bottlenecks, nor produce too much waste, it is important to study the coordination model of transmission and distribution grid, which is of great significance to improve the coordination degree between the various parts of the power grid. In China, the voltage level of the power grid is often by different levels of power grid enterprises or power supply business planning, managed by different departments. All levels of power grids should support each other, coordinate and optimize the planning, ensure the reliability and economy of the power grid, and avoid the problems of capacity mismatch between different voltage levels and unbalanced power supply. In the context of China's comprehensive construction of a strong smart grid with ultra-high voltage (UHV) grid as its backbone and coordinated development of power grids at all levels, it is a trend to speed up the coordination of power grids at all levels. At present, a few literatures have studied the coordination of transmission and distribution network (Duan and Yu, 2002; Zhang et al., 2010; Feng et al., 2016), and there is no literature report on the transmission network planning in line with distribution network. Smart grid has a crucial and beneficial role in ensuring network security in clean energy generation and improving power utilization efficiency. A large number of smart grid technology research and engineering construction work has been carried out at home and abroad, which provides a technical basis for conducting DR research (Habib, 2017).

\section{KEY SCIENTIFIC CHALLANGES}

\section{The Flexible Planning Method of Network Source Coordination and AC/DC Transmission Network Considering the Temporal and Spatial Distribution Characteristics of High Proportion Renewable Energy Sources}

When the ratio of non-water renewable energy exceeds a certain percentage, the structure of the power system will change from quantitative to qualitative change. The power system will appear the power balance probability, the power system operation diversification, the power flow two-way, Power system stability mechanism complexity, power system flexibility resource scarcity, transmission and distribution system source and scope limits fuzzy and other characteristics (Kang and Yao, 2017). Renewable energy share refers to the proportion of renewable energy generating capacity to the total electricity load; for the power base, renewable energy accounted for the proportion of renewable energy generation accounted for the total amount of local power generation. Under the combined effects of strong fluctuation and randomness at the source end and large load carrying load at the load terminal, the planning and operation characteristics of the transmission and distribution network will also undergo fundamental changes. In particular, for the source and the charge end, it is often necessary to predict 
Table 1. Comparison of Transmission grid planning methods

\begin{tabular}{|c|c|c|c|c|c|c|c|c|}
\hline \multirow[t]{2}{*}{ Planning method } & \multicolumn{2}{|l|}{ New grid form } & \multicolumn{3}{|c|}{$\begin{array}{l}\text { Object of } \\
\text { study }\end{array}$} & \multicolumn{3}{|c|}{$\begin{array}{c}\text { Planning } \\
\text { model }\end{array}$} \\
\hline & $\begin{array}{l}\text { High proportion } \\
\text { of renewable } \\
\text { energy }\end{array}$ & $\begin{array}{c}\text { AC and } \\
\text { DC } \\
\text { hybrid }\end{array}$ & $\begin{array}{l}\text { power } \\
\text { supply }\end{array}$ & $\begin{array}{c}\text { Transmission } \\
\text { network }\end{array}$ & $\begin{array}{l}\text { Distribution } \\
\text { network }\end{array}$ & $\begin{array}{l}\text { Random } \\
\text { planning }\end{array}$ & $\begin{array}{c}\text { Robust } \\
\text { planning }\end{array}$ & $\begin{array}{c}\text { Multi - } \\
\text { objective } \\
\text { programming }\end{array}$ \\
\hline $\begin{array}{l}\text { Network source collaborative } \\
\text { planning }\end{array}$ & $\checkmark$ & $\checkmark$ & $\checkmark$ & $\checkmark$ & & $\checkmark$ & & $\checkmark$ \\
\hline $\begin{array}{l}\text { Transmission network random } \\
\text { planning }\end{array}$ & $\checkmark$ & $\checkmark$ & & $\checkmark$ & & $\checkmark$ & & $\checkmark$ \\
\hline $\begin{array}{l}\text { Transmission network robust } \\
\text { programming }\end{array}$ & $\checkmark$ & $\checkmark$ & & $\checkmark$ & & & $\checkmark$ & \\
\hline $\begin{array}{l}\text { Distribution network in line with } \\
\text { the transmission network planning }\end{array}$ & $\checkmark$ & $\checkmark$ & & $\checkmark$ & $\checkmark$ & $\checkmark$ & & $\checkmark$ \\
\hline
\end{tabular}

the medium and long term and short term renewable energy output and load, which provides the corresponding boundary conditions for the planning and operation of the power system, while the renewable energy output is more uncertain about the time and space coupling; In addition, distributed renewable energy access to the "Net Load" forecast also brought great challenges; System operation mode diversification, decentralization, complexity, how to make the transmission network planning to accommodate all the running state, but also has the economy, are the power grid planning is facing important scientific problems. A flexible planning method of network source Coordination Planning and AC / DC transmission network planning is required to consider the high proportion of renewable energy sources, so as to adapt to the strong uncertainty of multiple time and space.

AC/DC Transmission Network Planning Scheme for Adaptive Energy Generation and Grid Operation Characteristics, Multi-Dimensional Fine Operation Simulation and Comprehensive Evaluation Method

Future high proportion of renewable energy grid will change the power system operating characteristics fundamentally. At the same time, with the development of multi-terminal DC and DC power grid, the future of AC / DC hybrid power grid to the power system will bring a series of planning and key technical issues such as topological structure, economic evaluation, safety evaluation, reliability evaluation and optimization. At present, the operation and planning of transmission network are mainly aimed at AC power grid. How to consider the characteristics of AC / DC hybridization in the operation and planning of transmission network is the focus of research. In particular, the incorporation of DC power grid will inevitably lead to changes in the overall topology of the grid, while incorporating a large number of power electronic equipment, its control strategy and operational characteristics will have an important impact on the operation of the grid. Power flow calculation method of AC/DC hybrid power grid, all kinds of constraints are different. (Cao et al., 2016) economic evaluation, security assessment and reliability evaluation need to establish a suitable mathematical model for power electronic equipment in power system. In the new grid form with high proportion of renewable energy and grid-connected AC-DC hybrid, the multi-dimensional fine operation simulation and comprehensive evaluation method of transmission network planning scheme are the key problems to be solved and solved urgently.

\section{MAIN LINE OF STUDY}

For high proportion of renewable energy and grid, AC and DC hybrid new grid form, this paper presents a study of transmission grid planning theory considering high proportion of renewable energy, Which is divided into the following four directions: (1) network source collaborative planning considering the spatial and temporal distribution of high proportion of renewable energy; (2) flexible planning of transmission grid based on stochastic programming and robust optimization; (3) Transmission network planning coordinated with distribution network; (4) Comprehensive evaluation and decision-making method of planning scheme. The first three points are the study of the transmission grid planning method, and the last point is the comprehensive evaluation method of the planning scheme. Different transmission network planning methods and their focus are shown in Table 1.

\section{MULTI-OBJECTIVE PROGRAMMING METHOD FOR AC/DC TRANSMISSION NETWORK}

\section{Network Source Collaborative Planning}

The output of wind power, photovoltaic and other renewable energy sources has the characteristics of strong volatility, randomness and time series. The massive power generation scene is directly integrated into the network 


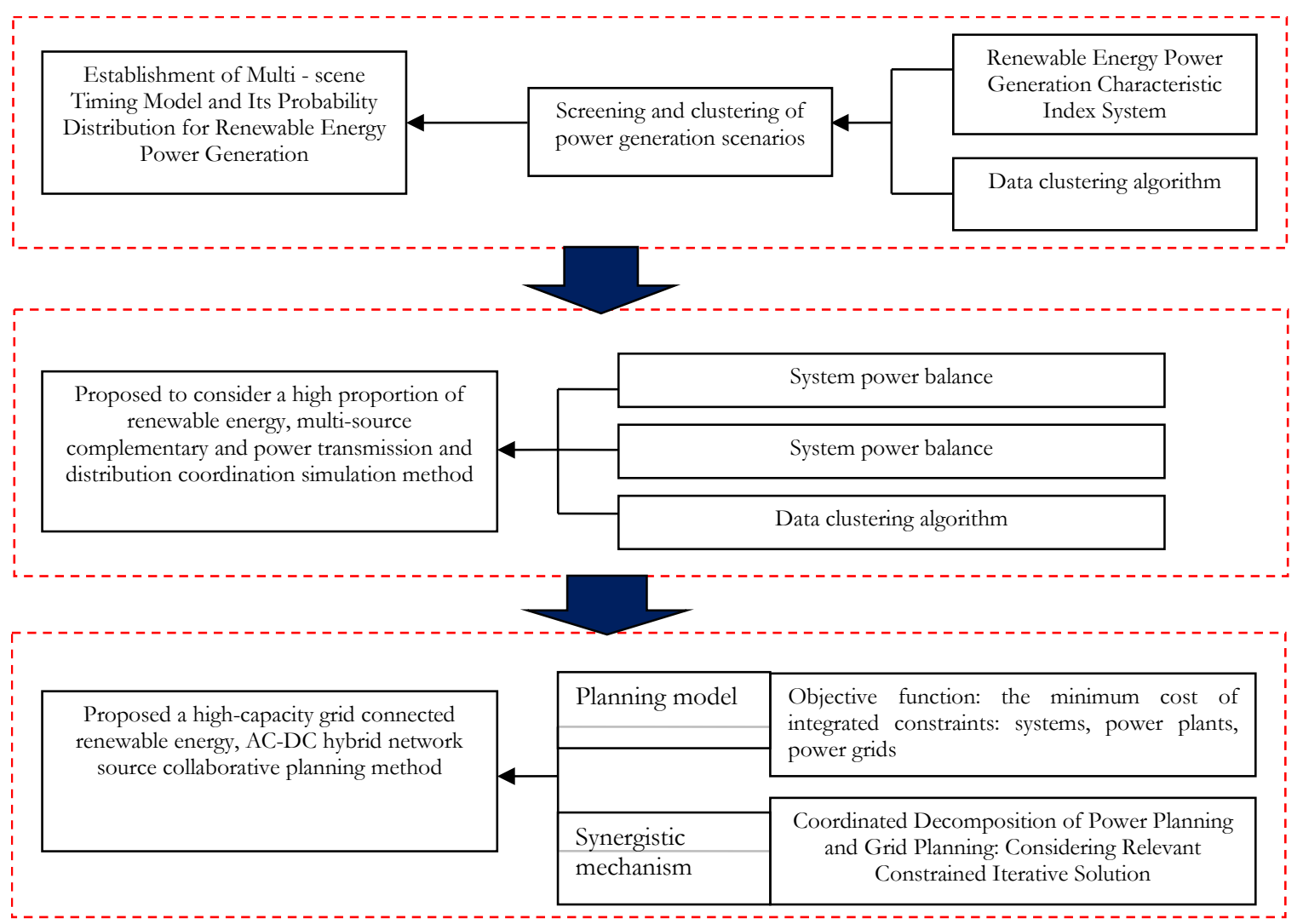

Figure 1. Research framework for Network source collaborative planning

source planning, and the workload of calculation is enormous. Based on the clustering method of massive power generation scene, the classification of power generation scene can be reduced, and the scale of network collaborative planning can be greatly reduced, but it is easy to omit the key scenes that have great influence on the system's power balance and peaking balance. Therefore, the need to consider the renewable energy power generation scene and load coupling characteristics, this paper presents a screening and clustering method for clustering scene and key scenes of renewable energy power generation, and establishes a multi - scene timing and probability distribution model for renewable energy power generation.

The basic task of running simulation is to objectively simulate the operation mode of the planned annual power system, verify the feasibility of the network resource collaborative planning scheme, and calculate the relevant economic and technical indicators. High proportion of renewable energy and the network will bring complex, multi-dimensional, uncertain operation of the power system. It is necessary to study a new method for coordinated operation of network sources to adapt to the high proportion of grid connected renewable energy, and requirements of mass solution evaluation for collaborative planning of AC / DC hybrid network sources. In addition, the modeling of network source coordination planning should take full account of the characteristics of high volatility and randomness of the high proportion renewable energy power generation, as well as the economic, security and environmental protection of the power system. Therefore, the cooperative mechanism of network source planning is the focus of the model optimization. A grid synergy research framework considering high proportion renewable energy is shown in Figure 1.

\section{Transmission Network Flexible Planning}

\section{Stochastic Planning of Transmission Network based on Multi-Scene Technology}

The main idea of the stochastic programming of transmission network based on multi-scene technology is to use uncertain factors as random variables with probability distribution, the scene set is generated by the discrete value process. The original uncertainty optimization problem is transformed into the deterministic optimization problem under the scene set. The key lies in the creation and reduction of scene sets, as well as the planning model and solution. In the new type of transmission network, with the increase in the proportion of renewable energy grid, uncertain factors doubled, the number of scenes will be exponentially grow. In order to solve the problem of sudden increase in the number of scenes, according to their thinking method the current researchers put forward a number of random planning in the scene reduction technology, it can be divided into the following two categories. 
(1) According to the clustering, its mathematical principles can be simply understood as by comparing the "distance" between the different scenes, thus merging the similar scene. This method has a relatively complete theoretical basis in mathematics, but it does not make full use of the nature and characteristics of stochastic optimization problems, thus it becomes relatively independent of stochastic optimization.

(2) According to the fruit clustering, this method uses the information of the optimization problem to cut the scene, for example, the reduction of the objective function based on the single scene problem is reduced by measuring the influence of various scenes on the objective function value, and the representative scene is reduced under the stochastic optimization problem. Compared to the strict mathematical derivation by clustering method, according to the results clustering scenario reduction method makes better use of the information of the original problem, but the lack of solid theoretical basis, belong to a kind of method of empirical and heuristic.

Considering the difference between scenes and the particularity of optimization problems, scene reduction is mainly divided into two steps: scene clustering and scene selection (Hong et al., 2016). K-mean value, Kcore/center, Class and fuzzy clustering are mainstream clustering methods. The main idea is to choose the most probabilistic scene. Rates of extreme events may be excluded, and the scenario will be more robust to delegates when appropriate extreme scenarios are retained. Scene selection studies need to evaluate whether the existing scene with the highest probability is selected or whether all the scenarios are combined into one of the most representative scenes based on multiple fields.

The optimal variables of the transmission network planning are the line investment decision variables and the system operation variables. The objective function generally includes the line investment cost and the system running cost. Constraints include investment constraints and system operational constraints. System operation constraints generally only consider the economic scheduling model, regardless of the unit start and stop state constraints. In the system operation constraint, it is necessary to establish the line flow constraint considering the line investment state. At the same time, the large $\mathrm{M}$ method is used to transform the constraints into linear constraints. Then, the whole planning model is transformed into a Mixed Integer Linear Programming (MILP) model.

\section{Robust Planning of Transmission Networks with High Proportion of Renewable Energy and Grid Connection}

Transmission network robust programming is a transmission network planning method based on robust optimization theory. Unlike stochastic programming, robust optimization describes uncertainties through indeterminate sets (Wei et al., 2013), and does not require probability distribution information for uncertainties. The design and construction of the uncertain set has important influence on the accuracy and complexity of the model.

The robust planning model of transmission network is usually the three layer optimization model: The upper objective function is the minimum investment cost and operating cost, and the decision variable is the linear integer variable of the line investment to determine the investment scheme of the line; The intermediate objective function is the largest operating cost to determine the worst operating scenario under the upper level investment scheme. The decision variables are new energy output and uncertain load, or other uncertain factors; the lower-level problem is the optimal power flow problem. The objective function is the minimum running cost, and the optimal power flow in the worst scenario under the upper investment scheme is determined.

The existing robust transmission network planning method in renewable energy output and load forecast uncertainty based on interval model characterization, but did not consider the relationship between renewable energy output correlations and inter node load. Therefore, we need to consider the correlation of uncertain factors and establish a robust programming model that characterizes the correlation between renewable energy output and forecast load. Transmission grid flexible planning research framework shown in Figure 2.

\section{Transmission Network Planning in Coordination with Distribution Networks}

\section{Value Indicators}

The value indicators in transmission grid planning are usually measured by the functional relationship between cost and benefit (Zhang et al., 2014). This function can be either a difference between the two, or a ratio of the two. The value indicator derived from the difference relationship indicates the size of the net income, but it applies only to cases where the cost and benefit have the same dimension; the value derived from the ratio relationship indicates the yield of the unit cost. Although it is applicable to the case that the cost and the income dimension are inconsistent, the ratio relationship is easy to be introduced into the nonlinear, and the embedded model will increase the difficulty of solving the model. Lifecycle Cost (LCC) refers to all direct, indirect, derivative or derivative products that occur or are likely to occur in the product life cycle or the expected life cycle, product design, research investment, use, maintenance and product assurance. The sum of all the costs are non-derivative 

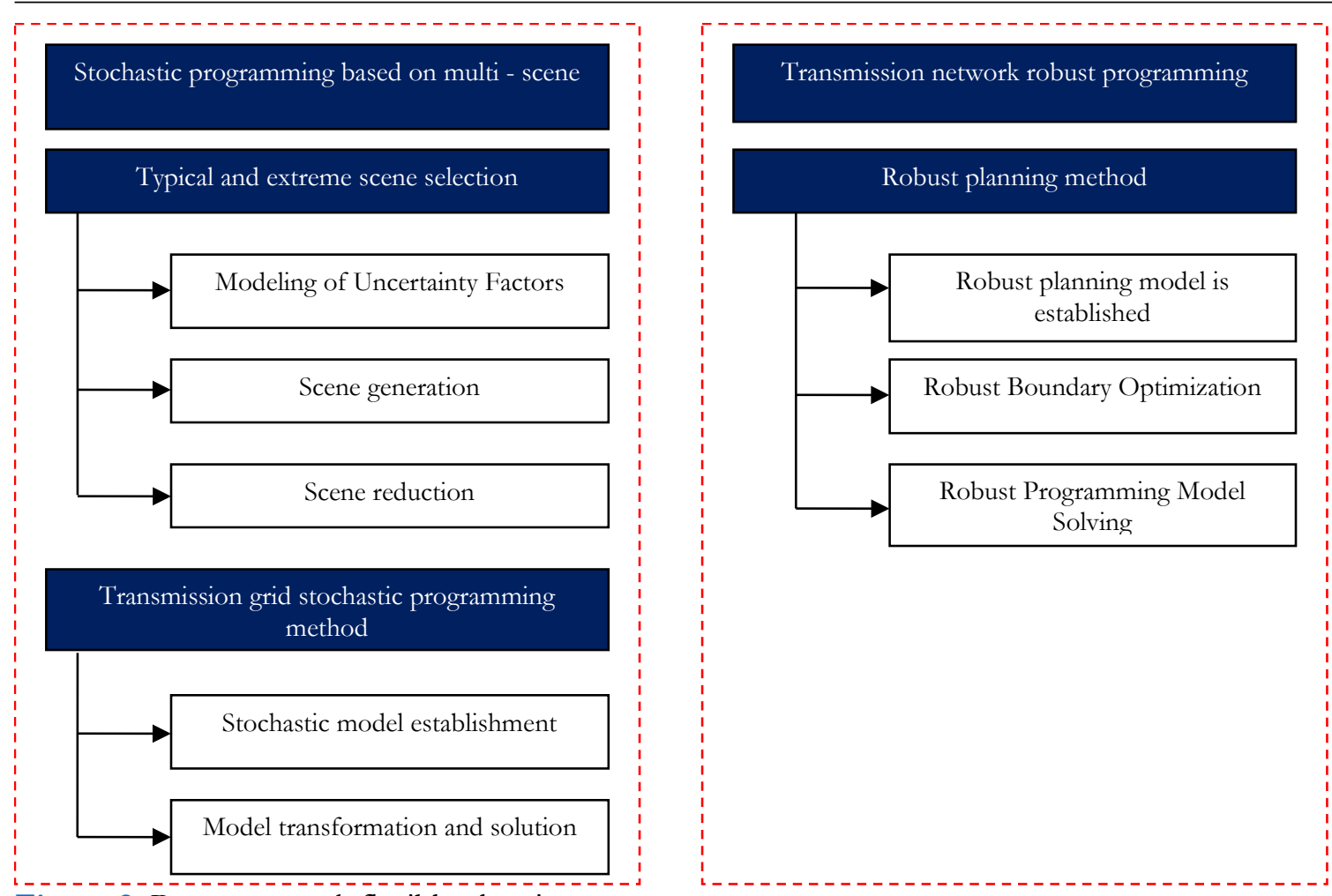

Figure 2. Power network flexible planning

(Cai et al., 2011). LCC is more accurate and comprehensive than traditional economic indicators (Liu et al., 2012a). In (Liu et al., 2012b) paper, a three-dimensional LCC hierarchical model including time dimension, component dimension and cost dimension is established for the transmission network, and the economic evaluation strategy of power system based on LCC is proposed. The difficulty and key of LCC modeling is the system cost structure or decomposition structure, which guarantees neither omission nor repetition. The existing LCC model only considers the equipment fault uncertainty, without considering intermittent renewable energy and other uncertain factors, so it is necessary to study the uncertainty of the transmission network specification scheme of LCC model under the environment of different factors (Zhang et al., 2016).

\section{Risk Indicators}

Risk assessment in power system is to identify the possibility of uncertainty, the possibility of accidents and the severity of the consequences, its essence is to further measure the uncertainty to achieve the risk of investment in the grid controllable (Tian et al., 2014). From the point of view of grid planning, the risks exist mainly in the feasibility and optimality of the planning scheme (Wang et al., 2009). There are technical risks in the feasibility of the planning scheme. The technical risk is caused by the uncertainty of the parameters required for the system simulation. It mainly involves the satisfaction of the planning and stability conditions in the future environment, such as the existence of power grid planning program for the safety and stability of the operation may be more limited. There are economic risks in the optimality of the planning scheme. The economic risk is due to the unpredictable market forecast and the uncertainties of the market behavior of the participants, but if the volatility of the quotation parameters in the market transaction makes the grid income uncertain.

Effective risk assessment relies on the construction of risk indicators, i.e., the description of risk. At present, the way of describing the risk in the power system is mainly divided into certainty and probability: One is the use of random variables such as the expected value and variance and other mathematical features; the other is the use of finance for risk (value at risk, VaR), management risk value and conditional risk value (conditional value at risk, CVaR) (Cao et al., 2014). The risk makes the process of grid planning more complicated, and it is an important research direction to avoid the risk of power grid planning through reasonable allocation.

\section{Transmission and Distribution Grid Coordination Indicators}

At present, the transmission and distribution network coordination assessment system has not yet been formed, which needs further study. Reasonable coordination evaluation of transmission and distribution network should be constructed from the aspects of reliability, economy and technology to construct the coordination index system of transmission and distribution network. In the various indicators of the evaluation system, it can be divided into transmission network index, distribution network index and transmission and distribution grid index, the first two are based on the transmission and distribution network as two separate research objects to build coordination 


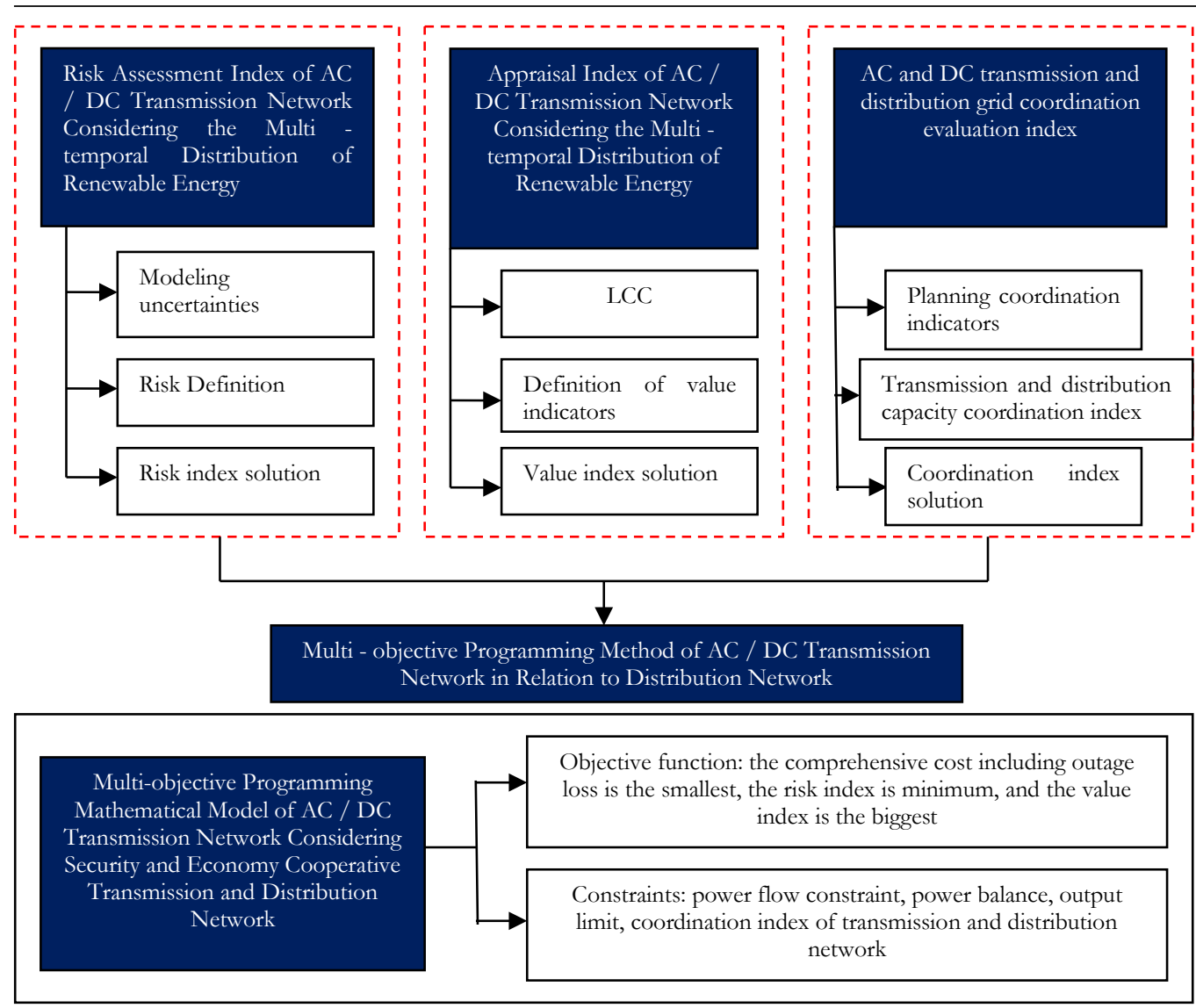

Figure 3. Multi-Objective Programming of AC / DC Transmission networks in coordination with Distribution Networks

indicators, while the latter is to build a coordination index with the transmission and distribution network as a whole. Indicators should have clear physical meaning and concise and clear mathematical expression, the relationship between indicators and screening is the need to focus on the content. The coordinated index system of the transmission and distribution network can also be used as a strong support for the definition of the coordination of the transmission and distribution network, and the two are complementary.

\section{Multi-Objective Programming of Transmission Networks in Coordination with Distribution Networks}

On the basis of value index, risk index and coordination index of transmission and distribution network, A multi objective programming method for AC / DC transmission network considering the economic and safety is developed. The technical route is shown in Figure 3.

\section{Comprehensive Evaluation and Decision Making of Planning Scheme}

In order to establish a power system operation simulation model considering network topology and renewable energy randomness, first of all, the influence of network constraints and peak shaving constraints on the abandoned power of high proportion renewable energy is analyzed, and a method of power system balance analysis based on fine operation simulation is put forward.

(1) Considering the temporal and spatial characteristics of renewable energy power generation, the time series output model of renewable energy base is established, and the renewable energy generation technology is proposed to meet the requirements of production simulation.

(2) The time series production simulation technique considering the mass operation scenario of renewable energy is put forward to improve the accuracy of power and power balance analysis.

(3) In order to meet the requirements of the simulation of the difference between the output of the renewable energy and the load characteristics in the high proportion of the multi - region, the paper puts forward the simulation method of the grid topology and the transmission capacity, the network constraint of energy dissipation for renewable energy and the precise calculation method of peak regulation constraints are put forward.

Analysis of effective and reasonable convergence of electrical power system operation simulation and calculation of power system planning, extraction to meet the high proportion of renewable energy consumption of the power grid safety operation margin is relatively small, Considering the characteristics of renewable energy 


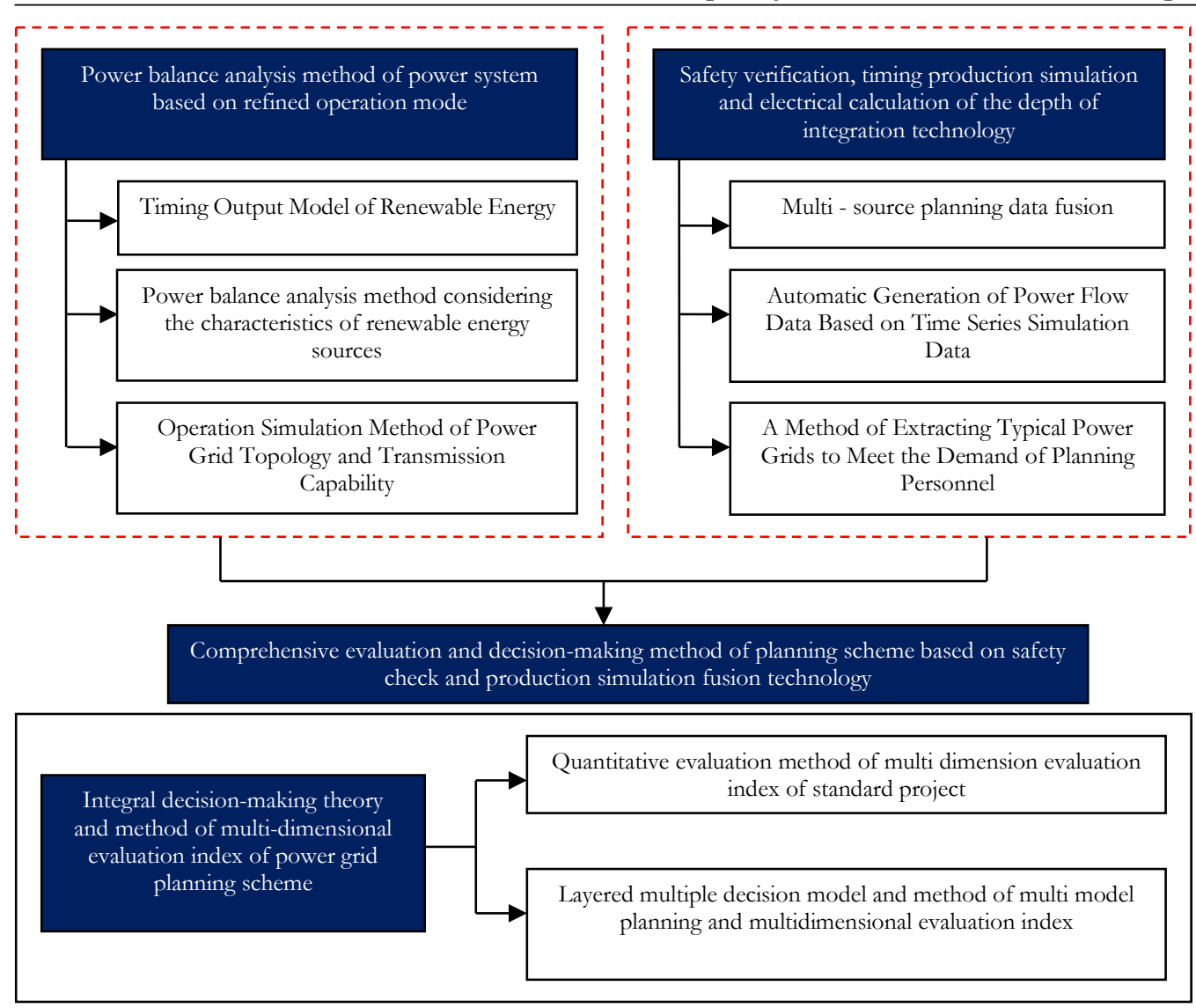

Figure 4. Based on security check and Production Simulation Fusion Technology Comprehensive Evaluation and Decision-making Method of Planning

output, it is necessary to propose a deep fusion technology for safety verification, time series production simulation and electrical calculation which adapts to the temporal and spatial distribution characteristics of high proportion renewable energy sources.

Specifically include:

(1) to establish a data model that meets the requirements of data fusion such as power load forecasting, power planning, grid planning and so on;

(2) the automatic generation of power flow data based on the simulation data of time series operation is proposed, HVDC is proposed to improve the convergence of the power flow planning in the power grid. In this paper, the convergence method of the power flow in the power grid is improved by considering the high proportion of renewable energy and high voltage direct current; (3) a typical method of grid extraction is proposed to meet the needs of planners. Finally, a comprehensive decision theory and method of grid planning scheme with multiple planning models and multidimensional evaluation indexes are constructed. The framework is shown in Figure 4.

\section{CHALLENGE AND OPPORTUNITIES}

\section{Difficulties and Solution of Network Resource Collaborative Planning}

Massive renewable energy power generation scene into the network source collaborative planning model, the calculation of a huge workload, the need to achieve through the clustering scene reduction. Conventional clustering methods tend to miss out on scenes with small probabilities and greater impact on power system operation. In the process of clustering and screening, consider the coupling characteristics of renewable energy power generation and power system load, the key scenes which influence the system power balance and peak shaving balance are increased, and the multi scene time series and probability distribution model of the renewable energy power generation is constructed together with the clustering scene.

The network source collaborative programming model is a large-scale, high-dimensional, non-linear mixed integer programming problem, and computational solution is more difficult. On the one hand, by studying the corresponding coordination mechanism, the large-scale network source planning problem is decomposed and decomposed to form a number of easy-to-solve sub-problems; On the other hand, we can study the high 
performance hybrid optimization method of network source collaborative planning model based on the intelligent optimization method with strong global search ability and the mathematical optimization method with high local optimization efficiency.

\section{Difficulties in Flexible Planning of Transmission Network and Solution}

Due to the uncertainty of factors such as renewable energy and load, the planning scheme developed by the conventional grid planning method is difficult to implement or has a large deviation. At present, the main challenge of transmission network planning based on multi-scene technology is: (1) how to consider the modeling and operation control mode of AC / DC power network; (2) although the use of scene reduction technology, but the planning model is still large scale, so the need to study the efficient planning model Solving technology. The establishment of robust programming theory is a difficult point in the study of power system, and there are not many research results at home and abroad, based on the study of robust optimization, robust control and robust scheduling of power system, the system theory of robust planning for power system is established.

In the robust planning of power system, the method of stochasticity or randomness of intermittent power supply is based on the probability model of intermittent power output. The system operating state is reduced to multiple scenarios (uncertain sets), and the mixed integer programming method is used to solve them. Model solution can be considered to make full use of CPLEX and other commercial mathematics optimization software for algorithm research. On the other hand, the game theory method of robust programming for power systems is also studied.

\section{Difficulties and Solutions to Transmission Network Planning in Relation to Distribution Network}

The AC / DC transmission network planning model, which is coordinated with the distribution network, is a complex large-scale nonlinear mixed integer programming model, and it is difficult to obtain the optimal solution. The modern heuristic optimization algorithm has some advantages in solving the nonlinear mixed integer programming model. The modern heuristic optimization algorithm and the original dual interior point method can be used to solve the planning model. The modern heuristic optimization algorithm is used in the planning scheme Formation and optimization; the original dual interior point method is used to verify the security of the planning model.

In addition, whether it is benefit or cost, the calculation depends to a great extent on the accuracy of the data. Data not only with time changes, but also with the social, economic development, policies are closely related, it is difficult to accurately obtain. In the cost part, LCC technology in the power system planning application is rare, its cost elements involved in many factors, the typical value of each factor is inconclusive. In the benefit section, the existing grid as a whole, the benefits of different voltage levels of the grid are difficult to distinguish.

In order to capture all kinds of state information and meet the needs of the application system, the sampling frequency of the apparatus is getting higher and higher. For example, in the state (China) monitoring system of power transmission and transformation apparatus, in order to diagnose the condition such as the insulation discharge, the sampling frequency of the signal must be above $200 \mathrm{kHz}$ (UHF detection needs GHz sampling rate). Thus, for a smart grid equipment-monitoring platform, the amount of data stored in the monitoring or testing is very large (Habib and Hafeez, 2017).

High proportion renewable energy grid connection will become an important feature of the future power system. However, the future power of high proportion renewable energy access in system, Wind power and solar energy as an important pillar of power supply, the temporal and spatial distribution characteristics and uncertainty will lead to power system operation mode change. So, its urgent to re-explore the power system planning and operation mechanism (China's National Bureau of Energy, 2016).

Study the value and impact of the cost elements in the LCC, while keeping up with the national, industry and enterprise policy standards. For example, in the scenario where the electricity market has not been fully liberalized and the sale price has not yet been differentiated, the optimal flow income model can be used to calculate the financial return, (according to the Opinions of the Central Committee of the Communist Party of China and the State Council on further deepening the reform of the electric power system) with the formal development, the calculation methods of power system benefit are greatly affected. Therefore, a new method to calculate the economic efficiency directly using electricity price is needed.

\section{Comprehensive Evaluation and Decision-making Difficulties and Solution of Planning}

Based on the simulation results of production operation, it is difficult to generate the power flow data with better convergence, which lays the data foundation for electrical calculation. In the large-scale renewable energy and network, the random characteristics of renewable energy determines the power system operation of the diversity, there is no significant typical scenes and extreme scenes. The traditional manual power flow adjustment method is difficult to adapt to the demand of mass flow mode analysis. Therefore, it is necessary to study the 
automatic generation of power flow based on the simulation results of production operation simulation. The technology of automatic generation of tidal current mainly converts the DC power flow data of the simulation results of production operation to the data needed by the special simulation software (such as BPA) for AC power flow calculation. Comparing the BPA AC power flow with the DC power flow input data, the main difference is that one is the reactive power flow calculation including reactive power balance, and the other one is the active power flow calculation without reactive power balance. Therefore, the automatic flow production technology aims at the DC power flow data of each production operation simulation operation result, according to the active power planning of each node and its power factor, etc., using node based square lattice method, the initial value of reactive power planning for power generation and load with reactive load nodes or equipment is calculated; On this basis, the reactive power balance of the system is calculated in the unbalanced situation, the initial value is adjusted and optimized according to the sensitivity coefficient. As the initial value of the reactive power output plan, the fusion of the simulation results of operation and the automatic generation of the power flow is realized.

At the same time, for the generation of automated batch generation, the ill conditioned power flow in power grid planning data is refined for the realization of batch AC flow with new energy sources and large-scale HVDC, Automatic calculation, put forward the solutions of the system, including the unreasonable flow data identification technology, the trend of auxiliary analysis technology, reactive power planning and regional adjustment of reactive power optimization technology, the convergence of calculation data effectively improve the trend of power grid planning.

\section{CONCLUSION}

In this paper, the scientific problems of transmission grid planning under the new grid form are put forward. Based on the research status in the world, the research direction and content of transmission grid planning with high proportion of renewable energy are discussed. Network source Coordination Planning Considering the temporal and spatial distribution characteristics of high proportion renewable energy resources, flexible planning of power transmission network based on stochastic programming and robust optimization, the research framework and technical route of transmission network planning are put forward from four aspects of transmission network planning, planning scheme, comprehensive evaluation and decision-making method. At the same time, the corresponding solutions are proposed to provide guidance and suggestion for the future research of grid transmission planning considering the high proportion of grid connected renewable energy.

\section{REFERENCES}

Aghaei, J., Amjady, N., Baharvandi, A., et al. (2014). Generation and transmission expansion planning: MILPbased probabilistic model. IEEE Trans on Power Systems, 29(4), 1592-1601. https://doi.org/10.1109/TPWRS.2013.2296352

Akbari, T., Rahimikian, A. and Kazemi, A. (2011). A multi-stage stochastic transmission expansion planning method. Energy Conversion $\&$ Management, $52 \quad$ (8/9), $2844-2853$. https://doi.org/10.1016/j.enconman.2011.02.023

Alvarez, L. J., Ponnambalam, K. and Quintava, V. H. (2007). Generation and transmission expansion under risk using stochastic programming. IEEE Trans on Power Systems, 22(3), 1369-1378. https:// doi.org/10.1109/TPWRS.2007.901741

Arslan, H., Luo, L. and Bilawal, A. (2017). Demand and Application of Energy Storage Technology in Renewable Energy Power System. American Scientific Research Journal for Engineering, Technology, and Sciences, 36(1), 75-84.

Cai, Y., Liu, L., Cheng, H. Z., et al. (2011). Application Review of life cycle cost (LCC) technology in power system. Power System Protection and Control, 39(17), 149-153.

Cao, J., Yan, Z., Li, J., et al. (2016). Probabilistic power Flow calculation for AC/DC hybrid system with wind farms. Electric Power Automation Equipment, 36(11), 94-101.

Cao, Y., Cao, L., Li, C., et al. (2014). A model and Algorithm for transmission expansion planning considering the blackout risk. Proceedings of the CSEE, 34(1), 138-145.

Carrion, M., Arroyo, J. M. and Alguacil, N. (2007). Vulnerability constrained transmission expansion planning: a stochastic Programming approach. IEEE Trans on Power Systems, 22(4), 1436-1445. https://doi.org/10.1109/TPWRS.2007.907139

Chen, B. and Wang L. (2016). Robust transmission planning under uncertain generation investment and retirement. IEEE Trans on Power Systems, 31(6), 5144-5152. https:// doi.org/10.1109/TPWRS.2016.2538960 
Chen, B., Wang, J., Wang, L., et al. (2014). Robust optimization for Transmission expansion planning: minimax cost vs. Minimax Regret. IEEE Trans on Power Systems, 29(6), 3069-3077. https://doi.org/10.1109/TPWRS.2014.2313841

Cheng, H., Fan, H. and Zhai, H. (2007). Review of transmission flexible planning. Proceedings of the CSUEPSA, 19(1), 21-27.

China's National Bureau of Energy. (2016). 2016 first half of the country's wind power grid operation.

Dehghana, S., Amjady, N. and Conejo, A. J. (2017). Adaptive robust Transmission expansion planning using linear decision rules. IEEE Trans on Power Systems. https://doi.org/10.1109/TPWRS. 2017.2652618

Ding, Y., Wang, P., Goel, L., et al. (2012). Long-term reserve Expansion of power systems with high wind power penetration using universal generating function methods. IEEE Trans on Power Systems, 26(2), 766-774. https://doi.org/10.1109/TPWRS.2010.2054841

Duan, G. and Yu, Y. (2002). Global optimization for power Transmission and distribution system planning. Proceedings of the CSEE, 22(4), 109-113.

Energy Research Institute of the national development and Reform Commission. (2015). China's 2050 high proportion renewable energy development scenario and Path Study.

Feng, Y., Yun, Z., Sun, J., et al. (2016). Fast situation Awareness method for distribution network coordinated with transmission grid. Automation of Electric Power Systems, 40(12), 37-44. https://doi.org/10.7500/AEPS20160311009

Gao, C., Wu T., He, Y., et al. (2012). Generation and Transmission coordinated planning considering wind power Integration. Automation of Electric Power Systems, 36(22), 30-35.

Guy, Mccalley, J. D. and Ni, M. (2012). Coordinating large-scale wind Integration and transmission planning. IEEE Trans on Sustainable Energy, 3(4), 652-659. https://doi.org/10.1109/TSTE.2012.2204069

Habib, A., Arshad, A. and Khan, R. (2017) Distributed Renewable Energy under the Guidance of Price Autonomous Operation Technology. Smart Grid and Renewable Energy, 8, 305-324. https://doi.org/10.4236/sgre.2017.810020

Habib, A., Sou, C. and Ananta, A. (2017). Control Strategy of DC Link Voltage Flywheel Energy Storage for Non Grid Connected Wind Turbines Based on Fuzzy Control. Journal of Power and Energy Engineering, 5, 72-79. https://doi.org/10.4236/jpee.2017.511006

Habib, A. (2017). Overview of Leading Methodologies for Demand Response in Smart Grid and Demand Response case overview in China and abroad. International Journal of Novel Research in Computer Science and Software Engineering, 4(1), 19-34.

Habib, A. and Hafeez, H. M. (2017). 21st Century Present Condition and Challenges Related to Large-Scale Data Processing in Smart Grid and Optional Framework for Large Data Storage and Analysis. International Journal of Novel Research in Computer Science and Software Engineering, 4(1), 35-46.

Hand, M. M., Baldwin, S., De Meo, E., et al. (2014). Renewable Electricity Futures Research. Colorado, USA: National Renewable Energy Laboratory.

Hemmati, R., Hooshmand, R. A. and Khodabakhshiana. (2013). Comprehensive review of generation and transmission expansion planning. IET Generation Transmission \& Distribution, 7(7), 955-964. https://doi.org/10.1049/iet-gtd.2013.0031

Hong, S., Cheng, H., Zeng, P. L., et al. (2016). Coordinate generation and transmission expansion planning with clusters of core cases. Automation of Electric Power Systems, 40(22), 71-76. https://doi.org/10.7500/AEPS20151129005

Huang, Y., Yang, J., Wen, F., et al. (2013). Transmission system planning made capability of qualating intermittent generation sources. Automation of Electric Power Systems, 37(4), 28-34.

Jabr, R. A. (2013). Robust transmission network expansion planning with thin renewable generation and loads. IEEE Trans on Power Systems, 28(4), 4558-4567. https:/ / doi.org/10.1109/TPWRS.2013.2267058

Kang, C. and Yao, L. Z. (2017). Key scientific issues and theoretical research framework for power systems with high proportion of renewable energy. Automation of Electric Power Systems. https://doi.org/10.7500/AEPS20170120004

Latorre, G., Cruz, R. D., Areiza, J. M., et al. (2003). Classification of publications and models on transmission expansion planning. IEEE Trans on Power Systems, 18(2), 938-946. https://doi.org/10.1109/TPWRS.2003.811168

Liu, L., Wang, H., Cheng, H. Z., et al. (2012). Economic Evaluation of power systems based on life cycle cost. Automation of Electric Power Systems, 36(15), 45-50.

Liu, L., Cheng, H., Zeliang, M. A., et al. (2012). Multi objective Transmission expansion planning promising life cycle Cost. Proceedings of the CSEE, 32(22), 46-54. 
Lu, Z. X, Li, H. and Qiao, Y. (2016). Power system flexibility planning and meeting offers high proportion of Renewable energy. Automation of Electric Power Systems, 40(13), 147-158. https://doi.org/10.7500/AEPS20151215008

Majidi-Qadikolai, M. and Baldick, R. (2016). Stochastic Transmission capacity expansion planning with special scenario selection for convert contingency analysis. IEEE Tran son Power Systems, 31(6), 4901-4912. https://doi.org/10.1109/TPWRS.2016.2523998

Orfanos, G. A. and Georgilakis, P. S. (2013). Transmission expansion planning of systems with increasing wind power integration. IEEE Trans on Power Systems, 28(2), 1355-1362. https://doi.org/10.1109/TPWRS.2012.2214242

Price Water House Coopers LLP (PwC), Potsdam Institute for Climate Impact Research (PIK), International Institute for Applied Systems Analysis (IIASA). (2014). 100\% renewable electricity: a roadmap to 2050for Europe and North Africa.

Roh, J. H., Shahidehpour, M. and Lei, W. (2009). Market-based generation and transmission planning with uncertainties. IEEE Trans on Power Systems, 24(3), 1587-1598. https://doi.org/10.1109/TPWRS.2009.2022982

Rouhani, A., Hosseini, S. H. and Raoofat, M. (2014). Composite generation and transmission expansion planning considering distributed generation. International Journal of Electrical Power \& Energy Systems, 29(4), 1592-1601. https://doi.org/10.1016/j.ijepes.2014.05.041

Ruiz, C. and Conejo, A. J. (2015). Robust transmission expansion Planning. European Journal of Operational Research, 242(2), 390-401. https://doi.org/10.1016/j.ejor.2014.10.030

Tan, L., Lin, J., Dai, S., et al. (2016). An improved light robust optimization model and its linear counterpart. Proceedings of the CSEE, 36(13), 3463-3469.

Tian, S., Cheng, H., Zeng, P., et al. (2014). Review of transmission planning for integration large clusters of wind power. Proceedings of the CSEE, 34(10), 1566-1574.

Wang, Y., Cheng, H., Hu, Z., et al. (2009). Multi objective Transmission expected value planning considering risk of overloading. Proceedings of the CSEE, 29(1), 21-27.

Wei, W., Liu, F. and Mei, S. (2013). Robust and economic Scheduling school for power systems: Part one Theoretical foundations. Automation of Electric Power Systems, 37(17), 37-43.

Yu, H., Chung, C. Y., Wong, K. P., et al. (2009). A chance constrained transmission network expansion planning method with consideration of load and wind farm decision ties. IEEE Trans on Power Systems, 24(3), 1568-1576. https://doi.org/10.1109/TPWRS.2009.2021202

Yuan, Y., Wu, B., Li, Z., et al. (2009). Flexible planning of transmission system with large wind farm based on multi scenario probability. Electric Power Automation Equipment, 29(10), 8-12.

Zhang, N., Hu Z., Zhou, Y., et al. (2016). Source grid- Load coordinated planning model and fuzziness. Automation of Electric Power Systems, 40(1), 39-44. https:// doi.org/10.7500/AEPS20150607002

Zhou, J., Yu, Y. and Zeng, Y. (2011). Heuristic optimization Algorithm for transmission network expansion planning with large-scale wind power integration. Automation of Electric Power Systems, 35(22), 66-70.

Zhao, C. and Guan, Y. (2013). Unified stochastic and robust unit commitment. IEEE Trans on Power Systems, 28(3), 3353-3361. https://doi.org/10.1109/TPWRS.2013.2251916

Zhang, Y., Liu, H., Jiang, J., et al. (2010). Research on evaluation index and method of coordinated Planning between main system and distribution network. Automation of Electric Power Systems, 34(15), 37-41.

Zhang, J., Zhu, Z., Liu, L., et al. (2014). Transmission network value considering large-scale wind power integration. Automation of Electric Power Systems, 38(19), 47-51. https://doi.org/10.7500/AEPS20131028007

Zhang, L., Cheng, H. Z., Zeng, P. L., et al. (2016). Transmission network planning approaches based on Judgment theories. Automation of Electric Power Systems, 40(16), 159-167. https:// doi.org/10.7500/AEPS20150330018 